\title{
Oronasal Fistula with Palatal Involvement Secondary to Cocaine Use
}

\author{
Ayesha Raza (Meds 2015) \\ Faculty Reviewer: Dr Ali Hilal, MBChB, FRCSC, AFSA, FABMS, FRCSI (Department of Otolaryngology)
}

\section{BACKGROUND}

Cocaine abuse is associated with serious systemic complications, affecting the cardiovascular and nervous systems by activating the sympathetic pathway. ${ }^{1}$ Vasoconstriction is a well-known local sympathetic effect of cocaine that may lead to ischemia, inflammation, ulceration and ultimately necrosis of the nasopharyngeal structures implicated by snorting cocaine. ${ }^{2}$ Nasal septal perforation is present in $4.8 \%$ of abusers, making it the most common complication of cocaine abuse. ${ }^{3}$ Chronic cocaine use may contribute to larger lesions and further destruction of the surrounding osteocartilaginous structures. Specifically, the destruction of nasal and midfacial bones and soft tissues can lead to a syndrome called cocaine-induced midline destructive lesion (CIMDL). The diagnosis of CIMDL requires at least 2 of the following clinical or radiologic findings: nasal septal perforation, lateral nasal wall destruction, and hard palate involvement. ${ }^{4}$ We describe a patient with a history of cocaine abuse and signs of CIMDL, with midfacial destruction of the nasal septum and hard palate creating an oronasal fistula. To the best of our knowledge, we believe this is the first case of CIMDL with oronasal fistula reported in Canada.

\section{CASE PRESENTATION}

A 60-year-old male was referred to us with a 1-month history of a "hole" in his hard palate that caused fluids to leak out of his nose when drinking. Prior to presentation, he had been using denture adhesive gel as a temporizing measure for the palatal defect. He also disclosed that he had a nasal deformity, which previous physicians had attributed to his long history of cocaine snorting. The oral and nasal lesions were not associated with bleeding, pain, B-symptoms, nasal obstruction or recurrent sinusitis. His past medical history was notable for asthma and hypertension. His social history revealed a lifetime of tobacco use and many years of chronic cocaine use.

On physical examination, the patient looked well and was in no acute distress. The nasal vestibules were intact bilaterally with no erosion of the columellar skin. Anterior rhinoscopy revealed complete loss of the cartilaginous nasal septum, without saddle nose deformity. Flexible nasopharyngoscopy showed extensive mucosal crusting, severe atrophy of the turbinates bilaterally but no polyps or suspicious growths. Examination of the oral cavity showed normal mucosa and dentition overall, with a single $1 \mathrm{~cm}$ perforation at the midline of the hard palate, communicating with the floor of the nasal cavity. The hard palate was quite soft in the area surrounding the perforation, suggesting bone loss of $2-3 \mathrm{~cm}$ in circumference.
The soft palate was intact and the posterior pharynx was normal, with no ulcerating or fungating growths. The remainder of the head and neck examination was unremarkable.

Computed tomography (CT) scan showed extensive bony erosion of the nasal septum and hard palate, with mucosal thickening of the maxillary and ethmoid sinuses. There was no evidence of orbital or intracranial extension of this disease. Nasopharyngeal swab was positive for Staphylococcus aureus. Biopsy of the nasal cavity mucosa showed non-specific granulation tissue with no evidence of malignancy or infection.

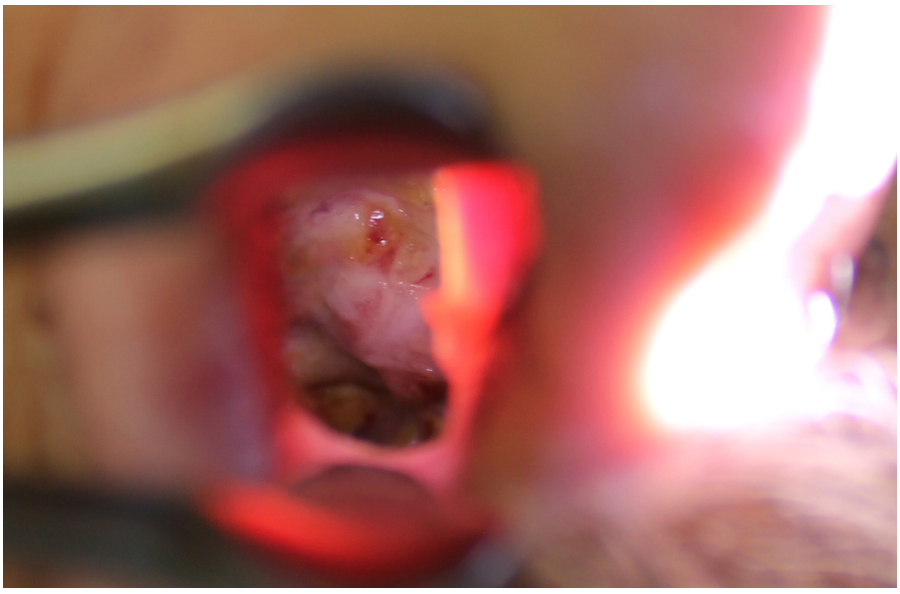

Figure 1. Anterior rhinoscopy showing a complete destruction of nasal septum and exposing the pale mucosa of the contralateral nasal cavity.

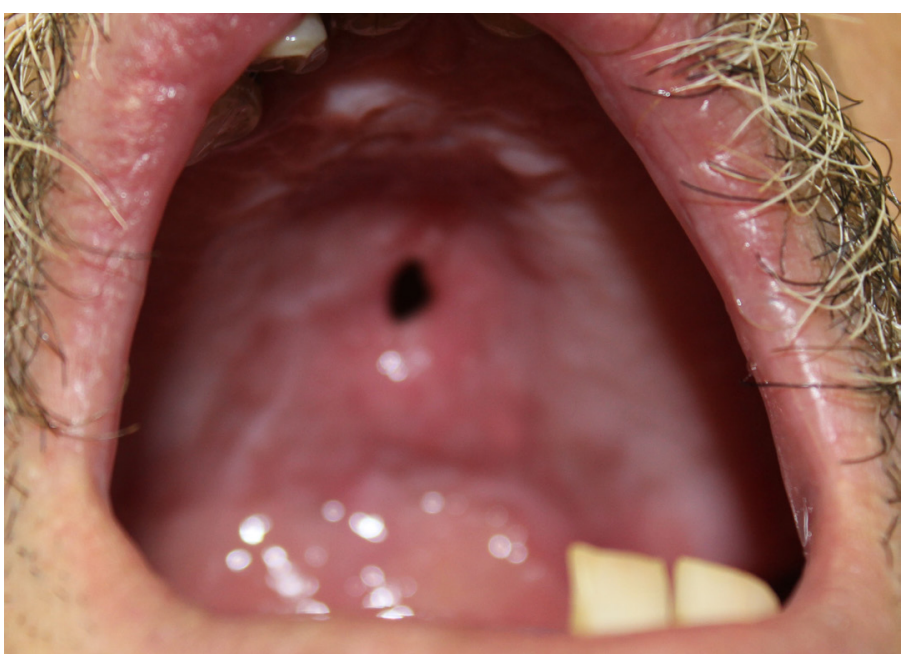

Figure 2. Photograph of hard palate with a midline oronasal fistula. 


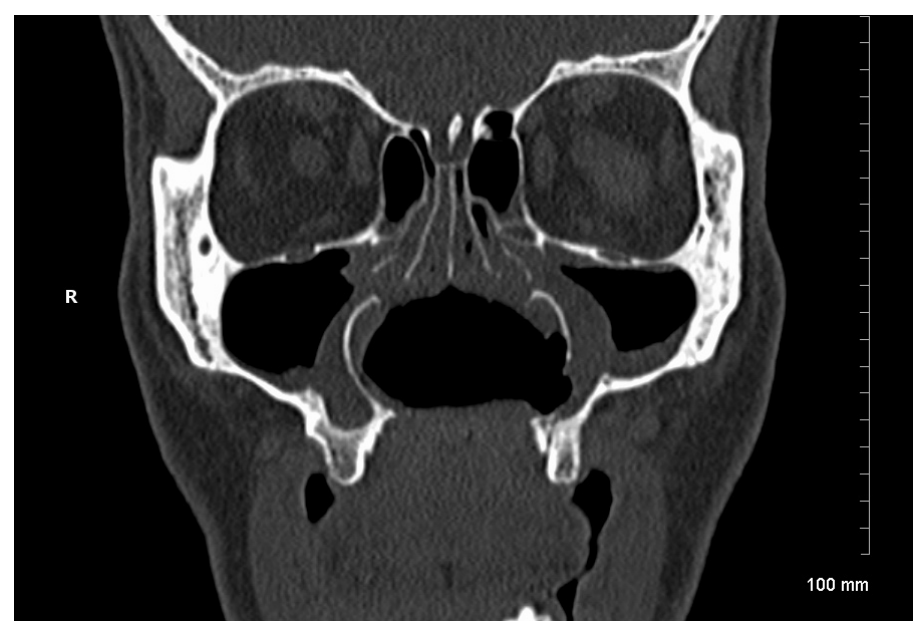

Figure 3. Coronal CT image of the nose and paranasal sinuses showing the complete loss of the nasal septum and turbinates.

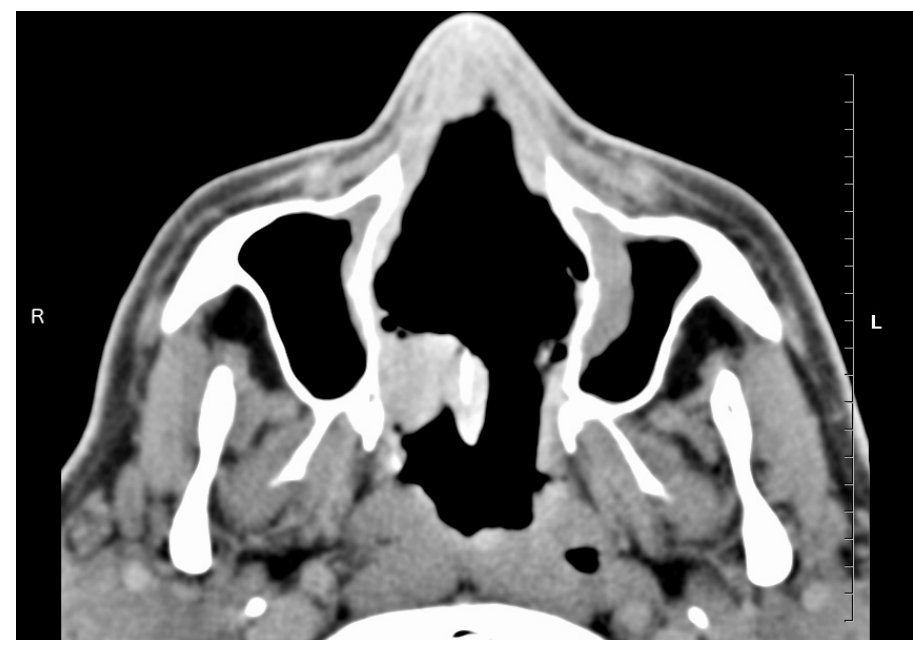

Figure 4. Axial CT image of the nasal cavity showing the soft tissue and bony loss of the nasal walls and septum.

We concluded that the patient's severe atrophic rhinitis and ischemic erosion of the nasal floor were due to years of intranasal cocaine use. Though the patient expressed interest in surgical closure of the perforation, the likelihood of a successful procedure is greatly reduced with a background of mucosal ischemia and continued local injury due to cocaine use. We discussed this with the patient and urged him to discontinue his drug use. He agreed to look into local addiction treatment programs with the help of his family physician. In the interim, we have advised frequent nasal saline rinses and referred him to an oral maxillofacial surgeon for fitting of a prosthetic obturator as a conservative measure for his oronasal communication. We felt that an obturator would stop his nasal regurgitation.

\section{DISCUSSION}

Though cocaine abuse has declined over the last decade, it remains among the top five most used substances among Canadian adults. ${ }^{5}$ Well-known systemic effects of cocaine use include tachycardia, hyperthermia and mydriasis. ${ }^{6}$ Snorting cocaine also causes local anesthesia and vasoconstriction, which predispose to trauma and mucoperichondrial ischemia of the nasopharyngeal system. Coupled with adulterant-induced mucosal irritation, these effects lead to erosion and destruction of the nasal septum. ${ }^{7}$ Septal perforations are the best-known external manifestations of cocaine abuse and are generally well tolerated, thus delaying abusers' medical presentation. However, continued local ischemic necrosis causes progression of nasal manifestations to severe midline destruction involving the palate, causing an oronasal fistula in a condition called CIMDL. The most commonly reported symptoms in patients with CIMDL are rhinolalia and the passage of liquids and food through the nose when swallowing-both of which finally prompt patients to seek medical attention. Anosmia, pain, headache and halitosis with cacosmia are also frequent presenting complaints. ${ }^{8}$

The treatment of CIMDL is challenging due to patients' non-compliance and hesitancy to admit to cocaine use. The first line of management is to exclude other potential causes for midline nasal destruction, with CT, lesion biopsy, blood tests and toxicology screen. When considering restorative therapy, the importance of abstaining from drug use must be stressed to patients, as the midline destruction will progress until they have completely stopped using. Management during rehabilitation is therefore conservative, including analgesia, antibiotic therapy, surgical debridement of necrotic tissue and saline lavage to prevent further destruction. ${ }^{9}$ The next treatment option is a prosthetic obturator, an oral device fitted to the patient's palate that artificially closes the oronasal fistula for temporary relief of symptoms. These can be adjusted to fit progressively widening fistulas, but their stability depends on the patient's dentition and palatal morphology. Nevertheless, some patients find adequate relief with an obturator and do not seek further therapy. Surgical reconstruction via local flap procedures is the ideal treatment and has been successful with non-cocaine related oronasal fistulas. However, the unpredictable vascularity of ischemic local tissues in CIMDL leads to a high risk of surgical failure and flaps may only be suited to small fistulas. To optimize outcomes, some authors have suggested that surgical reconstruction only be attempted after complete drug rehabilitation for at least one yeartherefore, treatment must include psychiatric assistance. ${ }^{10}$ 


\section{REFERENCES}

1. Cregler LL, Mark H. Medical complications of cocaine abuse. N Engl J Med. 1986;315(23):1495-1500.

2. Wang SH, Wang HW, Wang JY. Effects of cocaine on human nasal mucosa. Eur Arch Otorhinolaryngol. 1993;250(4):245-8.

3. Messinger E. Narcotic septal perforations due to drug addiction. JAMA. 1962;179:964-5.

4. Smith JC, Kacker A, Anand VK. Midline nasal and hard palate destruction in cocaine abusers and cocaine's role in rhinologic practice. Ear Nose Throat J. 2002;81(3):172-7.

5. Health Canada. 2013. Canadian Alcohol and Drug Use Monitoring Survey: summary of results for 2012 [Internet]. Available from: http://www.hc-sc.gc.ca/hc-ps/drugs-drogues/ stat/_2012/summary-sommaire-eng.php\#ref.

6. Vasica G, Tennant CC. Cocaine use and cardiovascular complications. Med J Aust. 2002;177(5):260-2.
7. Lancaster J, Belloso A, Wilson CA, McCormick M. Rare case of naso-oral fistula with extensive osteocartilaginous necrosis secondary to cocaine abuse: review of otorhinolaryngological presentations in cocaine addicts. J Laryngol Otol. 2000;114(8):630-3.

8. Silvestre FJ, Perez-Herbera A, Puente-Sandoval A, Bagán JV. Hard palate perforation in cocaine abusers: a systematic review. Clin Oral Investig. 2010;14(6):621-8.

9. Bains MK, Hosseini-Ardehali M. Palatal perforations: past and present. Two case reports and a literature review. Br Dent J. 2005;199(5):267-9.

10. Di Cosola M, Turco M, Acero J, Navarro-Vila C et al. Cocaine-related syndrome and palatal reconstruction: report of a series of cases. Int J Oral Maxillofac Surg. 2007;36(8):721-7. 